\title{
Prediction of Blood miRNA-mRNA Regulatory Network in Gastric Cancer
}

\author{
Mona Noohi ${ }^{1}$, Mojdeh Hakemi-Vala*2, Jamileh Nowroozi ${ }^{3}$, \\ Seyed-Reza Fatemi ${ }^{4}$, Mehrouz Dezfulian ${ }^{1}$
}

\begin{abstract}
Background: The aim of the study was to suggest a high specific and sensitive blood biomarker for early GC diagnosis.

Methods: the expression data of miRNAs and mRNAs were collected from the blood samples of the GC patients based on literature mining. Bioinformatics tools and databases (PANTHER, TargetScan, miRTarBase, miRDB, STRING, and Cytoscape) were used to predict the regulatory relationship. Subsequently, expression level of the selected miRNA was evaluated in the blood samples of gastritis patients to recognize the common miRNA between the GC and gastritis patients.

Results: Analysis of 40 target genes by MCODE (installed in Cytoscape software) indicated 4 hub genes (WWP1, SKP2, KLHL42, and FBXO11) as a significant cluster in the PPI network related to miR-21, with Node Score Cutoff: 0.2, Degree Cutoff: 2 and K-Core: 2. In addition, the miRNA RT-qPCR results showed that, the expression level of miR-21 was significantly higher in gastritis group compared to the healthy group $(\mathrm{p}<0.05)$.

Conclusions: the present study clearly demonstrated the increasing level of blood miR-21 among the gastritis patients infected by $H$. pylori. Therefore, the altered miRNAs, especially overexpression of onco-miRs, may identify a potential link between miRNAs and pathogenesis of the $H$. pylori-related complications.
\end{abstract}

Keywords: Blood Profiling, Gastric Cancer, H. pylori, Mir-21, Regulatory Network.

\section{Introduction}

Diagnosis of Gastric cancer (GC), as the fourth most common cancer worldwide, at the advanced stage is an important reason for its lower overall five-year survival rate and then being the second leading cause of cancer death. Moreover, chemotherapy and curative gastrectomy are known as the current standard treatments for GC in several countries with a low survival advantage (1). Therefore, an early-stage diagnosis can improve the prognosis and also reduce the mortality rate of gastric cancer. Consequently, scientists attempt to better understand the molecular biology at early disease stage of GC.

The high-throughput sequencing and transcriptomic analysis improve the global perspective on the RNA expression patterns in the pathogenesis as well as the progression of various diseases (2). MicroRNAs (miRNAs), as endogenous 20-24 nucleotide non-coding small RNAs, are considered as major players in the post-transcriptional regulation of gene expression, which are involved in metabolism, cell proliferation and death, development and 
other biological processes as well as cancer and infectious diseases (3). Accordingly, several miRNAs have been identified in human genome, and according to the bioinformatics studies, it is estimated that about $30-92 \%$ of human genes are regulated by miRNAs $(4,5)$. Moreover, there are many reports indicating the critical role of miRNA expression patterns for the initiation and progression of tumors (3). For example, in Gastric cancer, some miRNAs are downregulated as a result of a tumor suppressor while the others such as miR-155 and miR21 , are over expressed in gastric mucosa indicating to oncogenes (6). Notably, the miRNAs control the expression of their target genes and participate in both tumor suppression and tumor promotion. Therefore, different miRNA expression profiles between the cancer patients and normal subjects can leads to the proper diagnosis and accurate treatment of cancers (3).

Similar to other cancers, GC is resulted from a combination of environmental factors, genetic variations, and epigenetic alterations. In this regard, identifying these different factors and the relationship among them would be helpful for searching some more specific tumor biomarkers in gastric cancer. According to the epidemiological studies, Helicobacter pylori (H. pylori) infection is considered as the main environmental risk factor for GC at all ages that accounts for nearly $90 \%$ of the gastric cancer patients (7). The colonization of gastric tissue with $H$. pylori almost always leads to the acute and chronic gastritis (8), and also the patients with gastritis due to $H$. pyolri are at high risk for GC.

Recently, several considerable efforts have been devoted to the discovery of novel noninvasive biomarkers, especially blood, for the diagnosis of gastric cancer (9) as well as exploring the impact of regulatory networks that can be helpful to fulfil this aim. According to literature mining mir-21 is the most important miRNA with a high relation to gastric disease (10). The present study was aimed to report a miRNA and its target genes in blood, which suggest high specific and sensitive blood biomarker for the early stage of the gastric cancer diagnosis. In this manner, expression data of miRNAs and mRNAs in blood samples obtained from the GC patients in terms of the literature mining were collected to predict the regulatory relationship. Afterward, the expression level of the selected miRNA was evaluated in the blood samples of $H$. pylori infected patients with gastritis to recognize the common miRNA between the GC and gastritis patients, as an earlier biomarker for screening GC.

\section{Materials and Methods Study design}

The present study was performed in five steps as follows:

1. Literature mining

2. Functional classification and pathway analyses of the extracted genes

3. Selection of the significant miRNA based on the literature data

4. Prediction of the miRNA target genes and regulatory relationship

5. Comparison of the selected miRNA expression level in the blood samples of the healthy controls and gastritis patients.

The summary of the study is shown as flowchart in Figure 1.

\section{Literature Mining}

The data used in this study were obtained from several databases such as PubMed (http://www.ncbi.nih.gov/pubmed/) and Science Direct (http://www.sciencedirect.com). The original experimental studies that had been published up to December 2019 were included in this study. Also, the inclusion criteria were as follows: 1) Articles that had been reported miRNA and mRNA differential expression (DE) in whole blood as well as the serum and plasma samples, 2) Articles that compared the GC patients with healthy subjects, 3) Articles that evaluated the patients in both genders and different ages, and 4) Experimental Studies that were quantified at least once. Moreover, those review articles and bioinformatics articles with 


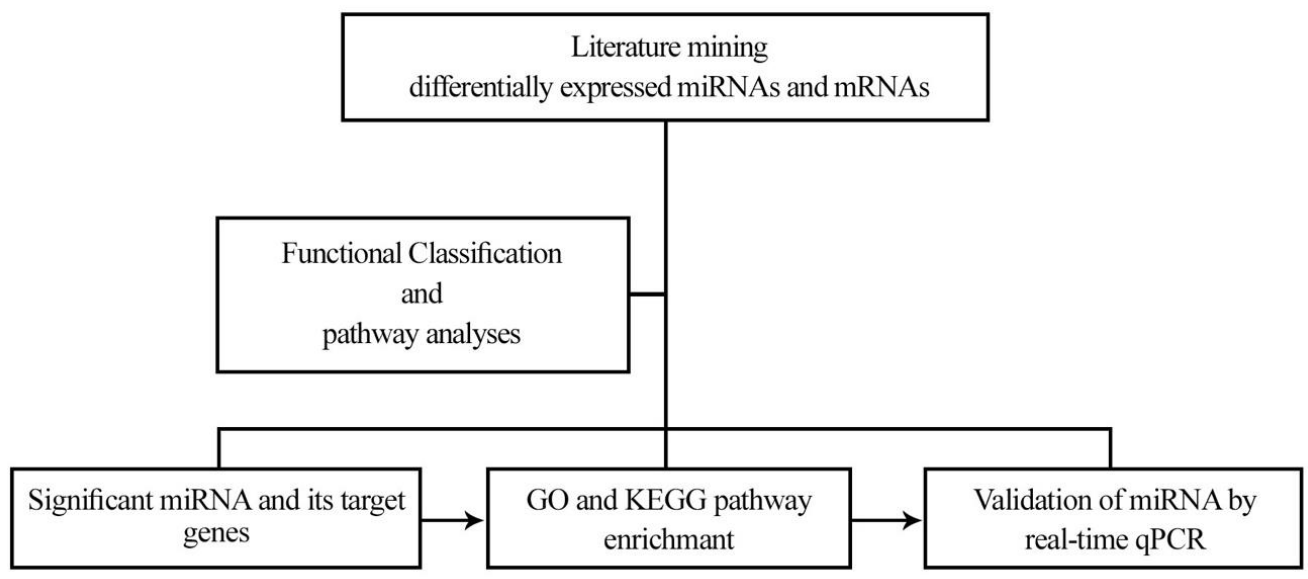

Fig. 1. Workflow of Study.

Table 1. Differential expressed mRNAs and miRNAs in blood sample of GC patients.

\begin{tabular}{|c|c|c|c|}
\hline Molecule & Method & Tissue & References \\
\hline $\begin{array}{l}\text { CRP, ORM1, PPBP, SEPP1, PGLYRP2, PON1, SAA2, } \\
\text { APOF, ADIPOQ, APOM, PF4, SERPINA3, LGALS3BP, } \\
\text { MST1, APOA1, C8G, HP, ANG, VTN, SERPING1, SAA1, } \\
\text { SERPINA7, HPX, A1BG, CFB, HBB, APOC3, HBD, } \\
\text { AHSG, PROZ, APOC1, C5, ITIH1, APOE, C1S, ITIH3, } \\
\text { LBP, CD14, HBA1, THBS1, CFHR1, CA1, ORM2, CFP, } \\
\text { SERPINA6, SERPINA1, CP, C4B, C6, C9, C4A, C2, SHBG, } \\
\text { S100A9, AFM, GPX3, LRG1, GC, HGFAC }\end{array}$ & $\begin{array}{l}\text { Detection: } \\
\text { MALDI-TOF mass } \\
\text { spectrometry } \\
\text { Validation: } \\
\text { LC-MS/MS }\end{array}$ & Serum & (11) \\
\hline $\begin{array}{l}\text { CDCP1, KAZALD-1, ADAMTS-15, DDAH-1, FLI1, MMP- } \\
\text { 10, CEACAM5, MMP-1, PPIA, MSLN, CCL20, APBB1IP, } \\
\text { TGF-alpha, SCF, ZBTB-17, HMOX-1, CA9, IL-7, IGF-1 }\end{array}$ & $\begin{array}{l}\text { Detection: } \\
\text { multiplex proximity extension } \\
\text { assays (PEA) (2) } \\
\text { Validation: } \\
\text { Elastic-net logistic regression } \\
\end{array}$ & Serum & (12) \\
\hline VTN, CLU, THBS1, SRMS & $\begin{array}{l}\text { Detection: } \\
\text { Quantitative Serum proteome } \\
\text { profiling } \\
\text { Validation: } \\
\text { MS-based quantitative analysis } \\
\text { (MRM-MS) }\end{array}$ & Serum & (13) \\
\hline NT5E, COPS2, TERF1, CTSF & $\begin{array}{l}\text { Detection: } \\
\text { human proteome microarray } \\
\text { Validation: } \\
\text { enzyme linked immunosorbent } \\
\text { assays (14) }\end{array}$ & Serum & (15) \\
\hline DENND1B, SMC1L1, PURB, PDCD & $\begin{array}{l}\text { Detection: } \\
\text { Affymetrix Gene Profiling } \\
\text { microarray } \\
\text { Validation: } \\
\text { A self-training logistic } \\
\text { regression model }\end{array}$ & $\begin{array}{l}\text { Peripheral } \\
\text { whole } \\
\text { blood }\end{array}$ & (16) \\
\hline hsa_miR_486, hsa_miR_451 & $\begin{array}{l}\text { Detection: } \\
\text { miRNA microarray } \\
\text { Validation: } \\
\text { real-time quantitative PCR }\end{array}$ & Plasma & (17) \\
\hline $\begin{array}{l}\text { hsa_miR_34, hsa_miR_17_5p, hsa_miR_744, } \\
\text { hsa_miR_376c, hsa_miR_27a, hsa_miR_20a, } \\
\text { hsa_miR_423_5p, hsa_miR_486, hsa_miR_451, } \\
\text { hsa_miR_106b, hsa_miR_378, has_miR_1, has_miR_21, } \\
\text { hsa_miR_106a, hsa_miR_221 }\end{array}$ & $\begin{array}{l}\text { Detection: } \\
\text { real-time quantitative PCR } \\
\text { Validation: } \\
\text { real-time quantitative PCR }\end{array}$ & Plasma & (18) \\
\hline
\end{tabular}




\begin{tabular}{|c|c|c|c|}
\hline hsa_miR_195_5p & $\begin{array}{l}\text { Detection: } \\
\text { real-time quantitative PCR }\end{array}$ & Plasma & $(19)$ \\
\hline hsa_miR_151_5p, hsa_miR_199a_3p & $\begin{array}{l}\text { Detection: } \\
\text { miRNA microarray } \\
\text { Validation: } \\
\text { real-time quantitative PCR }\end{array}$ & Plasma & $(20)$ \\
\hline $\begin{array}{l}\text { hsa_miR_486_5p, hsa_miR_16, hsa_miR_92a, } \\
\text { hsa_miR_25, hsa_miR_451 }\end{array}$ & $\begin{array}{l}\text { Detection: } \\
\text { TaqMan low density array } \\
\text { (TLDA) } \\
\text { Validation: } \\
\text { real-time quantitative PCR }\end{array}$ & Plasma & $(21)$ \\
\hline $\begin{array}{l}\text { hsa_miR_148a, hsa_miR_26a, hsa_miR_195, } \\
\text { hsa_miR_142_3p }\end{array}$ & $\begin{array}{l}\text { Detection: } \\
\text { miRNA Microarray and } \\
\text { TaqMan low density array } \\
\text { (TLDA } \\
\text { Validation: } \\
\text { real-time quantitative PCR } \\
\end{array}$ & Plasma & $(22)$ \\
\hline $\begin{array}{l}\text { hsa_miR_203, hsa_miR_92a, hsa_miR_181b, } \\
\text { hsa_miR_21, hsa_miR_31 }\end{array}$ & $\begin{array}{l}\text { Detection: } \\
\text { real-time quantitative PCR }\end{array}$ & Serum & $(23)$ \\
\hline $\begin{array}{l}\text { hsa_miR_18a, hsa_miR_92a, hsa_miR_19a, } \\
\text { hsa_miR_199a, hsa_miR_21 }\end{array}$ & $\begin{array}{l}\text { Validation: } \\
\text { real-time quantitative PCR }\end{array}$ & Serum & $(24)$ \\
\hline $\begin{array}{l}\text { hsa_miR_27a, hsa_miR_34, hsa_miR_423_5p, } \\
\text { hsa_miR_1, hsa_miR_20a }\end{array}$ & $\begin{array}{l}\text { Detection: } \\
\text { Solexa Sequencing } \\
\text { Validation: } \\
\text { real-time quantitative PCR }\end{array}$ & Serum & $(25)$ \\
\hline hsa_miR_378, hsa_miR_371_5p, hsa_miR_187 & $\begin{array}{l}\text { Detection: } \\
\text { genome-wide microRNA } \\
\text { microarray } \\
\text { Validation: } \\
\text { real-time quantitative PCR } \\
\end{array}$ & Serum & $(26)$ \\
\hline hsa_miR_376c, hsa_miR_744, hsa_miR_221 & $\begin{array}{l}\text { Detection: } \\
\text { TaqMan low density array } \\
\text { Validation: } \\
\text { Taq Man real-time quantitative } \\
\text { PCR }\end{array}$ & Serum & $(27)$ \\
\hline $\begin{array}{l}\text { hsa_miR_19a, hsa_miR_518f, hsa_miR_296, } \\
\text { hsa_miR_21, hsa_miR_30c, hsa_miR_223, hsa_miR_433, } \\
\text { hsa_miR_146a, hsa_miR_323_3p, hsa_miR_146b, } \\
\text { hsa_miR_451, hsa_miR_212, hsa_miR_19b, hsa_miR_106a, } \\
\text { hsa_miR_130a, hsa_miR_220, hsa_miR_374, hsa_miR_331, } \\
\text { hsa_miR_518d, hsa_miR_365 }\end{array}$ & $\begin{array}{l}\text { Detection: } \\
\text { TaqMan OpenArray assays } \\
\text { Validation: } \\
\text { TaqMan real-time quantitative } \\
\text { PCR }\end{array}$ & Serum & $(28)$ \\
\hline
\end{tabular}

Functional Classification and pathway analyses of the extracted genes

In this study, the PANTHER tool (Protein Analysis through Evolutionary Relationships, http://www.pantherdb.org) was used to classify the proteins of the extracted genes. Administering free available online resource, molecular function, biological process, cellular component classification, and pathway classification of the proteins are shown in pie and bar chart, respectively.
Figures 2 and 3 illustrate the PANTHER analysis.

\section{Selecting a significant miRNA based on the} literature data

Recent studies reported several circulating miRNAs, which could serve as a potential biomarker for GC. However, the identified mRNAs were not similar among different studies. At experimental stage, the miRNAs that had been reported more than others were selected. 
PANTHER GO-Slim Biological Process

Total * Genes: 85 Total * process hits: 89

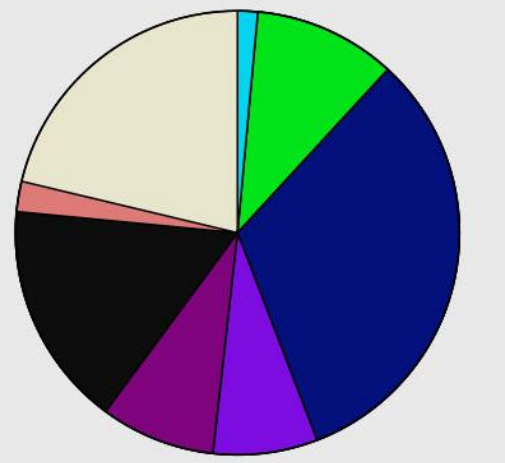

binding (GO:0005488)

catalytic activity (GO:0003824)

molecular function regulator (GO:0098772)

molecular transducer activity (GO:0060089)

structural molecule activity (GO:0005198)

transcription regulator activity (GO:0140110)

PANTHER GO-Slim Cellular Component Total * Genes: 85 Total * component hits: $\mathbf{5 0}$



cell (GO:0005623)

extracellular region (GO:0005576)

membrane (GO:0016020)

organelle (GO:0043226)

protein-containing complex (GO:0032991)

PANTHER GO-Slim Molecular Function Total * Genes: 85 Total * function hits: 80

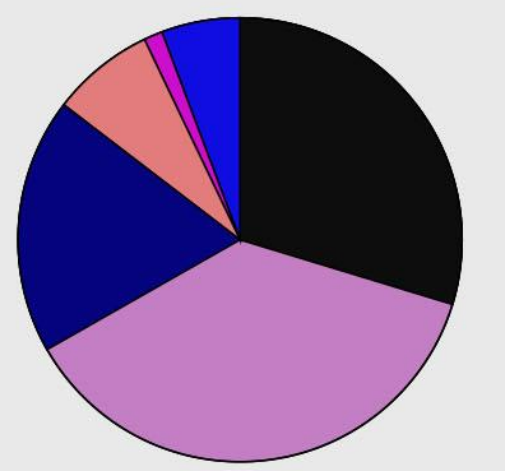

biological adhesion (GO:0022610)

biological regulation (GO:0065007)

cellular process (GO:0009987)

immune system process (GO:0002376)

localization (GO:0051179)

metabolic process (GO:0008152)

multicellular organismal process (GO:0032501)

response to stimulus (GO:0050896)

Fig. 2. Biological process, Molecular function and Cellular components of target genes were illustrated via PANTHER database Pie charts. 




Fig. 3. The pathway classification of target genes was illustrated via PANTHER database Bar chart. Blood coagulation, plasminogen activating cascade and Inflammation mediated by chemokine and cytokine signaling pathway allocated the most percentage of genes.

\section{Prediction of the miRNA target genes and regulatory relationship}

To predict the interactions between the selected miRNA and target genes, TargetScan (http:// www.targetscan.org), miRTarBase (http://miRTarBase.mbc.nctu.edu.tw/), and miRDB (http://www.mirdb.org) databases were used and solely genes were recognized by all three databases, which were considered as candidates. Moreover, a Search Tool for the Retrieval of Interacting Genes/Proteins (STRING) (http://string-db.org) was applied to construct a protein-protein interaction (PPI) network based on the identified genes (Fig. 4). Following the omission of the disconnected nodes, the PPI network was visualized using Cytoscape 3.7.0. (http://cytoscape.org/) as well as MCODE (Molecular Complex Detection) approach (installed in Cystoscope software) that was applied to screen the modules of hub genes from the PPInetwork. Final network is illustrated in Figure 5.
Comparison of the selected miRNA expression levels between the blood samples of the healthy controls and gastritis patients

Patients and samples

In this study, peripheral blood samples obtained from 50 patients (candidates for endoscopy who were diagnosed with dyspeptic symptoms by specialists), who were referred to Taleghani hospital (Taleghani hospital, Tehran, Iran) during 2017-2018, as well as the samples from 20 healthy volunteers (with no history of gastrointestinal diseases and taking no medications) were collected. Demographic characteristics of the patients are listed in Table 2. All the individuals signed the written informed consent form prior to participation in this study and it was also approved by ethical committees of Shahid Beheshti University of medical sciences with the number IR.SBMU.MSP.REC.1396.43. 


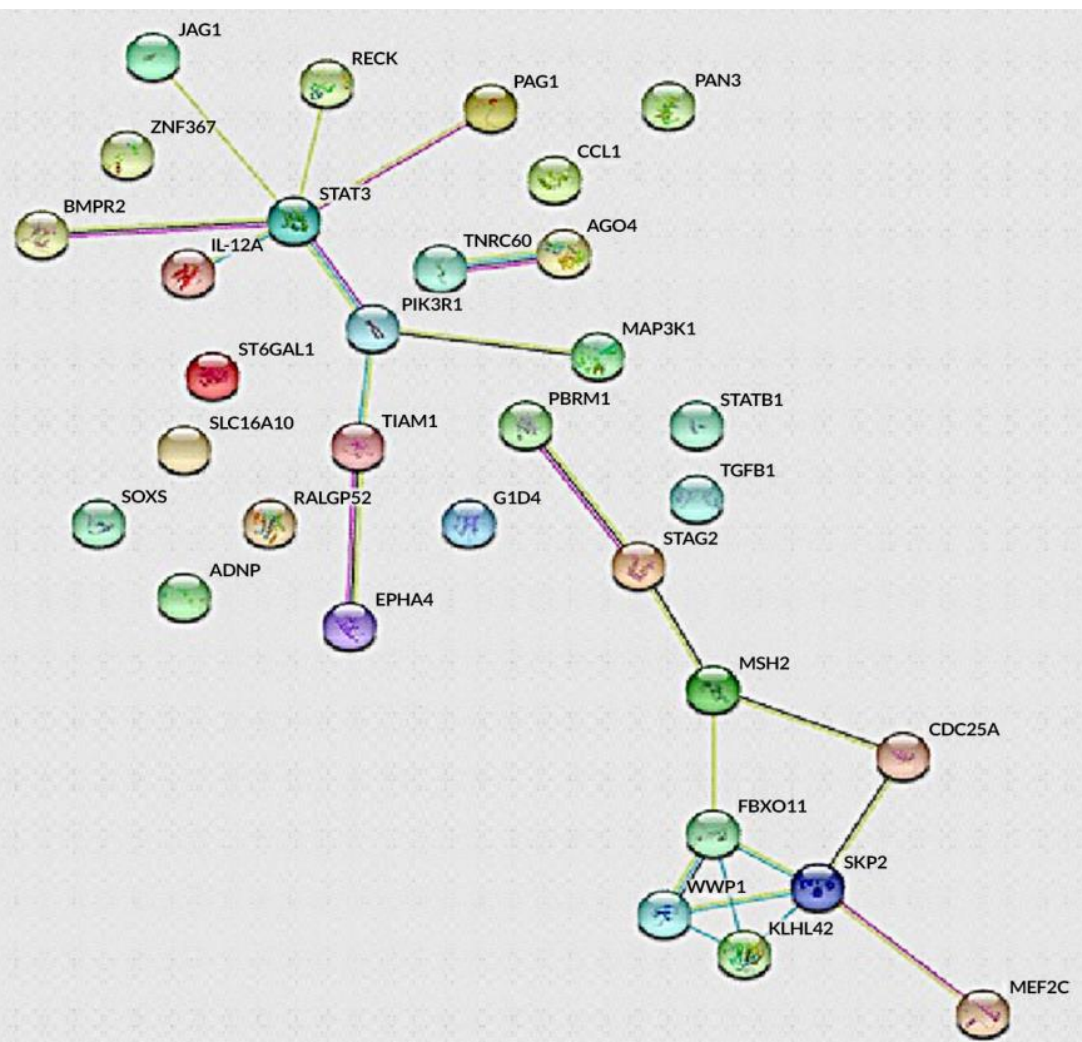

Fig. 4. PPI network of predicted miR-21 target genes, obtained from three databases and illustrated by STRING.

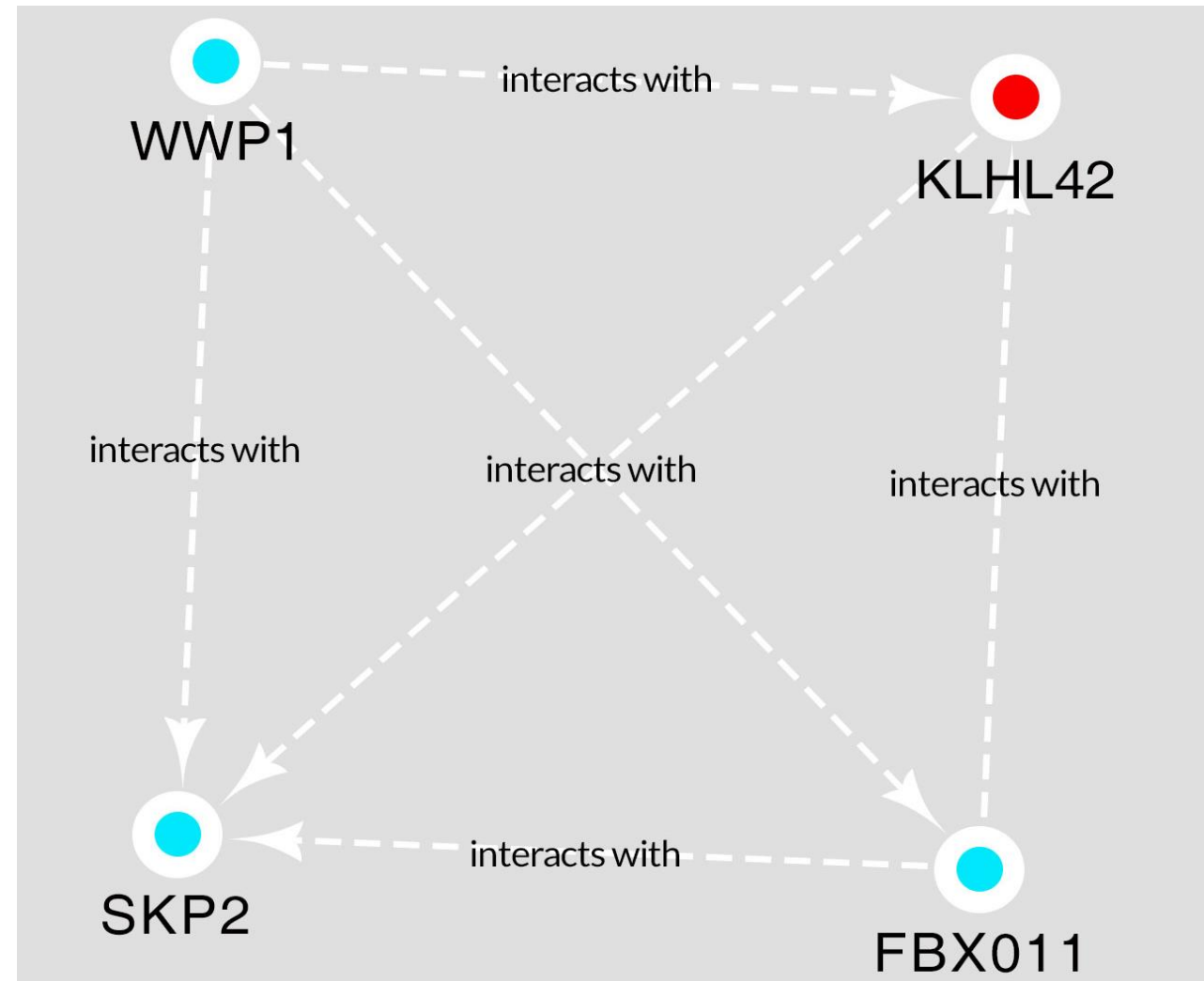

Fig. 5. Interactions of four hub genes extracted from the PPI network with the MCODE algorithm. The seed gene is illustrated in red color. 
Table 2. Demographic and clinical characteristics of patients and healthy individuals enrolled into the study.

\begin{tabular}{|c|c|c|}
\hline Characteristic & Gastritis patients & Healthy control \\
\hline Mean age & 18-83 (median48) & 17-80 (median43) \\
\hline \multicolumn{3}{|l|}{ Sex } \\
\hline Male/ Female & $23 / 27$ & $11 / 9$ \\
\hline Smokers & $15(30 \%)$ & - \\
\hline \multicolumn{3}{|c|}{ Canned food consumption } \\
\hline Non-consumer & $13(27.1 \%)$ & - \\
\hline Once in a month & $13(27.7 \%)$ & - \\
\hline Twice in a month & $6(12.9 \%)$ & - \\
\hline Once in a week & $8(15.7 \%)$ & - \\
\hline Twice in a week & $10(18.6 \%)$ & - \\
\hline \multicolumn{3}{|l|}{ Underlying disease } \\
\hline H. pylori infection & $33(65 \%)$ & - \\
\hline Heart diseases & $6(11.4 \%)$ & - \\
\hline Gastric diseases & $23(47.1 \%)$ & - \\
\hline Diabetes & $3(7.1 \%)$ & - \\
\hline Respiratory diseases & $5(10 \%)$ & - \\
\hline
\end{tabular}

\section{RNA Extraction, reverse transcription, and Real Time PCR}

To prepare the blood samples for RNA extraction, $500 \mu \mathrm{l}$ of fresh blood sample was drawn into EDTA tube and then centrifuged at $10000 \mathrm{rpm}$ for $2 \mathrm{~min}$ at room temperature. Afterward, the supernatant fluid was removed and $500 \mu \mathrm{l}$ RNase free water was added to precipitate, which was then centrifuged at $10000 \mathrm{rpm}$ for $2 \mathrm{~min}$ at room temperature, again. Subsequently, the supernatant fluid was removed and $1000 \mu 1$ miRNA Extractor solution was added to the precipitate. After this step, microRNA was isolated using Bio basic kit (Canada inc kit) in terms of the manufacturer's instruction. Also, DNase kit (deoxi-riobeuclease kit) was administered to remove any contamination of DNA from the extracted RNA, and immediately, the cDNA synthesis was performed using Maxime RT PreMix (oligo Dt primer, intron, Korea) in terms of the manufacturer's structure. The cDNA samples were then stored at $-80{ }^{\circ} \mathrm{C}$ until further processing. Detection of miR-21 was performed using Real Q Plus 2x Master Mix Green, no ROX (AmpliQon) Kit with standard protocol, which was carried out in a total volume $23 \mu$ l with $12.5 \mu$ l master mix cyber green NO ROX, $2 \mu$ forward: 5' GCTTATCAGACTGATGTTGA $3^{\prime}$ and reverse: 5' CAGCCCATCGACTGGTG 3' primers, $8.5 \mu \mathrm{l}$ of DEPC water. Moreover, the Real time program was done according to following program: initial denaturation for 5 min at $95{ }^{\circ} \mathrm{C}$, followed by 25 cycles with denaturation for $20 \mathrm{sec}$ at $95{ }^{\circ} \mathrm{C}$, annealing for $30 \mathrm{sec}$ at $60^{\circ} \mathrm{C}$, and extension for $20 \mathrm{sec}$ at 72 ${ }^{\circ} \mathrm{C}$. Real time data were analyzed using $2^{-\Delta \Delta C T}$ method (Livak and Schmittgen 2001). U6 was applied as a house keeping gene and miR-21.

\section{Statistical analysis}

All the statistical analyses were performed using SPSS 18.0 software (SPSS inc., Chicago, IL., USA) and data were presented as the mean \pm standard deviation. In addition, the Mann-Whitney test was used to compare the differences in the blood miR-21 concentrations between the gastritis group and the healthy control group. The correlation between miR-21 expression and type of gastric diseases as well as lifestyle habits questionnaire was analyzed using unpaired $t$ 
test. $\mathrm{P}$ value less than 0.05 was considered as statistically significant.

\section{Results}

Data mining, Functional enrichment, and pathway analyses

To better understand the DE of miRNAs and mRNAs and their interactions in the blood samples of the GC patients, the related data were gathered from 17 articles based on the present study's criteria. Accordingly, a total of 88 mRNAs and 50 miRNAs were identified (as there were limited studies focusing on the mRNA profiling in blood samples of GC, proteomics and gene profiling were also includes in the current study). The basic information of all the studies is listed in Table 1. Gene ontology classification was conducted to classify the blood proteins of the identified DEmRNA in blood samples of the GC patients. By applying the over representation test, the list of proteins was compared with a reference list in the PANTHER database ( 85 of 88 gene ID were matched by the reference list). Also, PANTHER was used for the classification of the obtained proteins through combining gene function, ontology, and pathways. Finally, the hit proteins in biological process were allocated to cellular process, molecular function allocated to catalytic activity, cellular component allocated to extracellular region, and pathways allocated to blood coagulation, and plasminogen activating cascade and Inflammation were mediated by chemokine and cytokine signaling pathways. The results are indicated in colorful pie and bar charts (Figs. 2 and 3).

\section{Construction of PPI network and module analysis}

Totally, 40 candidate genes were recognized based on searching in three databases (TargetScan, miRTarBase, and miRDB) that their protein-protein interaction was constructed and then analyzed by STRING (p-value were adjusted using the false discovery rate (FDR) method) (Fig. 4, Table 3). The resulted PPI network was analyzed using MCODE, indicating the hub genes as a significant cluster in the PPI network with the Node Score Cutoff: 0.2, Degree Cutoff: 2 and K-Core: 2. This module contains 4 nodes (WWP1, SKP2, KLHL42, and FBXO11) and 6 edges, in which KLHL42 was identified as seed node (Fig. 5).

Table 3. KEGG and Reactome pathways enrichment analysis of miR-21 target genes (Top 5).

\begin{tabular}{llcc}
\hline Pathway ID & Pathway term & Gene count & FDR \\
\hline KEGG pathways & & & \\
\hline hsa05206 & MicroRNAs in cancer & 4 & 0.0131 \\
\hline hsa05200 & Pathways in cancer & 6 & 0.0131 \\
\hline hsa04062 & Chemokine signaling pathway & 4 & 0.0131 \\
\hline hsa05418 & Fluid shear stress and atherosclerosis & 3 & 0.0340 \\
\hline hsa05203 & Viral carcinogenes & 3 & 0.0340 \\
\hline Reactome & & & \\
\hline HSA-166520 & Signaling by NTRKs & 4 & 0.0072 \\
\hline HSA-9006934 & Signaling by Receptor Tyrosine Kinases & 6 & 0.0120 \\
\hline HSA-8986944 & Transcriptional Regulation by MECP2 & 3 & 0.0120 \\
\hline HSA-8984722 & Interleukin-35 Signaling & 2 & 0.0120 \\
\hline HSA-8948700 & Competing endogenous RNAs (ceRNAs) & 2 & 0.0120 \\
\hline & & &
\end{tabular}


miRNA-21 expression level in the blood samples of the gastritis patients

The miRNA RT-qPCR experiments were performed on the blood samples of the gastritis patients in comparison with the healthy controls. Accordingly, the results showed that, the expression level of miR-21 was significantly higher in the patient group compared to the healthy group $(\mathrm{p}<0.05)$ (Fig. 6). Thus, the obtained data confirmed that, miR-21 expression was significantly up regulated in the gastritis samples (mostly infected by $\mathrm{H}$. pylori, 65\%) compared to the healthy samples.

\section{miR-21}

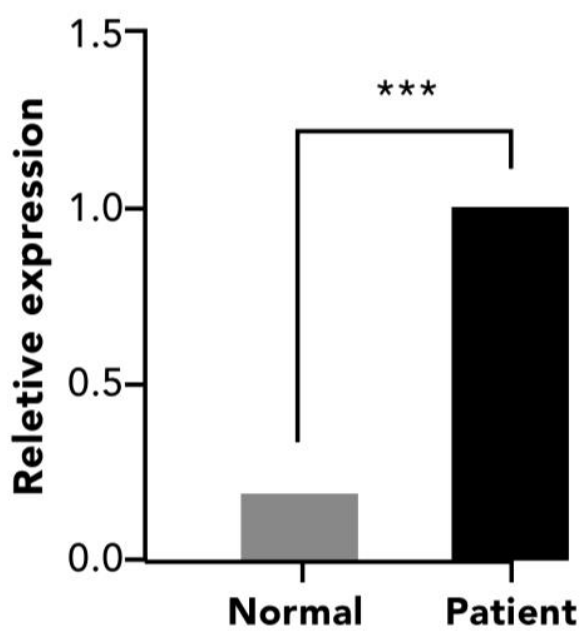

Fig. 6. Comparative RT-qPCR experiments of miR-21 level in patient and healthy samples. The level of U6 was used as internal control for normalization of miRNA expression. Data are reported as mean value \pm SE. Student's t-test was used for data analysis. $* * *$ Represents $\mathrm{p}$ value $<0.05$.

\section{Discussion}

Many subgroups of GC have been classified based on the histological types, molecular subtypes, and anatomical location. Also, due to the complex origins of histogenesis, pathogenesis, and molecular biology in tumor tissue; none of these classifications can accurately describe the potential molecular events that can trigger the origin of the tumor growth and invasion phenotype. In this regard, dysregulation of many miRNAs have been extensively documented in various diseases in recent years $(10,29)$. Moreover, many reports have revealed the specific alterations in the expression levels of several miRNAs in human gastric cancer compared to non-cancerous adjacent tissue $(30,31)$.

In recent studies, the circulating miRNAs have been investigated as the biomarkers for cancers on a miRNA microarray, also diagnostic and prognostic value of blood (plasma/serum) miRNAs in various cancers including GC have been demonstrated by many researchers. Although most of the studies that assessed the blood levels of miRNAs were selected based on miRNA profiling data in cancer tissues, some reports indicated the circulating miRNAs derived from tumor-cell lysis, and active secretion as a miRNA-protein complexes and/or in the form of cell-derived microvesicle. Consequently, the expression patterns in blood and other body fluids would not be identical with those of the cancer cell lines and tumor tissues (17). As a result of this issue, the first aim of the present study was to be focused on the experimental data from GC based on the blood samples to predict the GC miRNA-mRNA regulatory network in blood. To fulfil this, all the original articles up to December 2019, which had reported differentially expressed miRNAs and mRNAs in whole blood as well as in serum and plasma, 
performed between the GC patients and healthy subjects were extracted.

At the second stage of the study, a total of 88 genes from the literature mining were analyzed by PANTHER database, which indicated that, inflammation mediated by chemokine and cytokine signaling pathways has the highest score in this collection (Fig. 3). Among 50 miRNAs obtained from the literature mining, miR-21 had been reported more than other miRNAs (4 out of 11 studies), which was selected to explore for gene targets as well as regulatory network prediction.

MiR-21 is a known as onco-miR that is consistently and obviously upregulated in various human cancers. Accordingly, it targets many known tumor suppressors, and its overexpression by the inhibition of apoptosis and increasing cellular proliferation, shifts the balance between proliferation and apoptosis. Phosphatase and tensin homolog (PTEN) is a tumor suppressor, which is involved in both proliferative and apoptotic pathways. PTEN, which negatively regulates PI3K/Akt signaling pathway, is known as a specific target of miR21. Also, mutations in this gene are important in the progression of gastric carcinoma and many other cancers. Notably, programmed cell death protein 4 (PDCD4) is also targeted by miR-21. In proliferating cells, PDCD4 is localized to the nucleus; however, the direct role of this gene in apoptosis has not been elucidated yet. So, miR21 probably target PDCD4, which indirectly inhibits the apoptosis (32). In the current study, 40 target genes for miR-21 were identified using TargetScan, miRTarBase, and miRDB databases that none of them were similar to the 88 genes, which were expressed in blood profiling of GC (as mentioned earlier, Table 1). The KEGG and Reactome pathways enrichment analysis of miR-21 target genes indicated that, microRNAs in cancer, pathways in cancer, chemokine signaling pathway, and competing endogenous RNAs (ceRNAs) regulate PTEN translation are some of the significant enriched pathways of the target genes (Table 3).

Although the correlation between chronic bacterial infections and alteration in the expression level of miRNAs is a challenging issue for researchers, some authors recently reported that, $H$. pylori can affect the expression of various miRNAs, which may mediate epigenetic mis regulation of oncogenes and tumor suppressor genes and may also illustrate the bridge between $H$. pylori infection and gastric cancer $(10,29)$. MiR-21, as the first miRNA, was reported to be affected by $H$. pylori infection, which was found to be upregulated in both gastric cancer and $H$. pylori-infected tissues $(6,33)$. Therefore, the experimental stage of the present study was designed to evaluate the miR-21 expression level in the blood samples of the gastritis patients, which about $65 \%$ of them were $H$. pylori infected in comparison with healthy people. Moreover, the results indicated that, miR-21 was significantly overexpressed in the gastritis samples compared to the normal samples (Fig. 6).

Inflammatory cytokines and NF- $\mathrm{kB}$ could mediate the activation of AP-1 transcription, which are able to induce miR-21 and also explain miR-21 overexpression during $H$. pylori infection (34). In a study conducted by Liu et al., the expression of miR-21 in sporadic gastric tumor tissues was found to be remarkably upregulated as in non-cancerous tissues (35). In this regard, Shiotani et al. reported a higher expression of miR-21 in metaplastic gut mucosa compared with non-metaplastic mucosa, and that $H$. pylori eradication improves miRNA deregulation in the gastric mucosa, but not in metaplastic glands. They suggested that the Long-term colonization of $H$. pylori might induce the epigenetic modifications, which are not completely reversible by $H$. pylori eradication alone (6). A low expression of p27 (Cyclin-dependent kinase inhibitor 1B) has been reported in many cancers, including GC as well as in $H$. pylori infection, and c-Myc, as a transcriptional repressor of p27, is known as a target of Skp2. Kim et al. in 2006, investigated the effect of eradicating $H$. pylori on p27, cMyc, and Skp2 in mRNA and protein levels in chronic gastritis. Accordingly, the results indicated that, H. pylori downregulates p27 through increasing c-Myc in association with 
the increased expression of Skp2 (36). Also, in the current study, MCODE algorithm identified Skp2 as one of four genes, (WWP1, SKP2, KLHL42, and FBXO11) as a significant cluster of miR-21 target genes (Fig. 5). Therefore, more studies are needed for understanding the possible pathways mediated by Skp2 that may provide a mechanistic link between $\mathrm{H}$. pylori and gastric carcinogenesis.

The main limitation of the current study was that it was a case-control study. So, a prospective cohort study should be performed to support a causal relationship among $H$. pylori infection, gastritis incidence, and miRNA expression with the subsequent risk of gastric cancer development. Another limitation was that there were less experimental reports on DEmRNAs according to blood profiling of gastric cancer to approve the target genes of miR-21. Therefore, further in vitro and in vivo evaluation are required to achieve a comprehensive understanding role of $H$. pylori pathogenesis in misregulation of blood miRNAs and the associated abnormalities in cellular signaling in gastric cancer.

In conclusion, it should be noted that, $H$. pylori can alter the expression of various miRNAs in blood profiling of the gastritis patients. The present study clearly demonstrated

\section{References}

1. Leal M, AssumpÇÃo P, Smith M, Burbano R. Searching for Gastric Cancer Biomarkers Through Proteomic Approaches. Journal of Gastroenterology and Hepatology Research. 2014; 3:989-995.

2. Liu B, R Yuan, Z Lianga, T Zhanga, M Zhua, $\mathrm{X}$ Zhanga, et al. Comprehensive analysis of circRNA expression pattern and circRNAmRNA-miRNA network in Ctenopharyngodon idellus kidney (CIK) cells after grass carp reovirus (GCRV) infection. Aquaculture. 2019;512:734349.

3. Shu L, Wang Z, Wang Q, Wang Y, Zhanget X, et al. Signature miRNAs in peripheral blood monocytes of patients with gastric or breast cancers. Royal Society Open Biology. 2018;8(10). the increasing level of blood miR-21among the gastritis patients infected by $H$. pylori. Therefore, the altered miRNAs, especially overexpression of onco-miRs, may identify a potential link between miRNAs and pathogenesis of the $H$. pylori-related complications. However, how the dysregulation of these miRNAs is involved in the pathological development of gastric cancer is not quite clear yet. As a matter of fact, high throughput sequencing should be used to obtain the complete descriptions of blood miRNA misregulation in the $H$. pylori-related diseases, which would also be helpful to introduce a noninvasive and prognostic blood biomarker for early diagnosis and/or screening of high-risk population for gastric cancer.

\section{Acknowledgements}

The present article is a part of an accepted research plan and is financially supported by "Research Department of the School of the medicine, Shahid Beheshti University of Medical Sciences; SBMU" (Grant No. 8975) and is approved by ethical committees of Shahid Beheshti University of Medical Sciences (Ethical No IR.SBMU.MSP.REC.1396.43).

4. Miranda KC, Huynh T, Tay Y, Ang Y, Tam W, Thomson AM, et al. A pattern-based method for the identification of MicroRNA binding sites and their corresponding heteroduplexes. Cell. 2006;126(6):1203-17.

5. Xu P, M Guo, BA Hay. MicroRNAs and the regulation of cell death. Trends Genet. 2004;20(12):617-24.

6. Zhang Z, Li Z, Gao C, Chen P, Chen J, Liu $\mathrm{W}$, et al. miR-21 plays a pivotal role in gastric cancer pathogenesis and progression. Lab Invest. 2008;88(12):1358-66.

7. Jiang F, Shen X. Current prevalence status of gastric cancer and recent studies on the roles of circular RNAs and methods used to investigate circular RNAs. Cell Mol Biol Lett. 2019;24:53. 
8. Kusters JG, AH van Vliet, EJ Kuipers. Pathogenesis of Helicobacter pylori infection. Clinical microbiology reviews. 2006;19(3):449-490. 9. Li T, Shao Y, Fu L, Xie Y, Zhu L, Sun W. Plasma circular RNA profiling of patients with gastric cancer and their droplet digital RT-PCR detection. J Mol Med (Berl). 2018;96(1):85-96.

10. Zabaleta J, MicroRNA: a bridge from $H$. pylori infection to gastritis and gastric cancer development. Front Genet. 2012;3:294.

11. Abramowicz A, Wojakowska A, GdowiczKlosok A, Polanska J, Rodziewicz P, Polanowski $\mathrm{P}$, et al. Identification of serum proteome signatures of locally advanced and metastatic gastric cancer: a pilot study. J Transl Med. 2015;13:304.

12. Shen Q, Moghaddam M. A targeted proteomics approach reveals a serum protein signature as diagnostic biomarker for resectable gastric cancer. EBioMedicine. 2019;44:322-333.

13. Yoo, MW, et al. Discovery of gastric cancer specific biomarkers by the application of serum proteomics. Proteomics. 2017; 17(6).

14. Corder EH, GD Mellick. Parkinson's disease in relation to pesticide exposure and nuclear encoded mitochondrial complex I gene variants. J Biomed Biotechnol. 2006;2006:27601.

15. Yang L, Wang J, Li J, H Zhang, Guo S, Yanet $\mathrm{M}$, et al. Identification of serum biomarkers for gastric cancer diagnosis using a human proteome microarray. Mol Cell Proteomics. 2016;15(2):614-23.

16. Shi J, Cheng Ch, Ma J, Liew Ch, Geng X, et al. Gene expression signature for detection of gastric cancer in peripheral blood. Oncol Lett. 2018;15(6):9802-9810.

17. Konishi H, Ichikawa D, Komatsu S, Shiozaki A, Tsujiura M, H Takeshitaet, et al. Detection of gastric cancer-associated microRNAs on microRNA microarray comparing pre-and post-operative plasma. $\mathrm{B} \mathrm{j}$ cancer. 2012;106(4):740-747.

18. Cai H, Yuan Y, Hao Y, Guo T, Wei X, Zhang $\mathrm{Y}$, et al. Plasma microRNAs serve as novel potential biomarkers for early detection of gastric cancer. Med Oncol. 2013;30(1):452.

19. Gorur A, Balci Fidanci S, Dogruer Unal N, Ayaz L, Akbayir S, Yildirim H, Yaroglu Y, et al. Determination of plasma microRNA for early detection of gastric cancer. Mol Biol Rep. 2013;40(3):2091-6.

20. Li C, Fang Li J, Cai Q, Qiu Q, Yan M, B Ya Liu, et al. miRNA-199a-3p in plasma as a potential diagnostic biomarker for gastric cancer. Ann Surg Oncol. 2013;20 Suppl 3:S397-405.

21. Zhu C, Ren C, Han J, Ding Y, Du J, Dai N, et al. A five-microRNA panel in plasma was identified as potential biomarker for early detection of gastric cancer. $\mathrm{Br} \mathrm{J}$ Cancer. 2014;110(9):2291-2299.

22. Qiu X, Zhang J, Shi W, Liu S, Kang M, Chu $\mathrm{H}$, et al. Circulating microRNA-26a in plasma and its potential diagnostic value in gastric cancer. PloS one. 2016;11(3):e0151345.

23. Huang S, et al., Serum microRNA expression profile as a diagnostic panel for gastric cancer. Jpn J Clin Oncol. 2016;46(9):811-818.

24. Liu HN, Wu H, Tseng Y, Chen Y, Zhang D, Zhu L, et al. Serum microRNA signatures and metabolomics have high diagnostic value in gastric cancer. BMC cancer. 2018;18(1):415.

25. Liu R, Zhang C, Hu Z, Li G, Wang C, Yanget $\mathrm{C}$, et al. A five-microRNA signature identified from genome-wide serum microRNA expression profiling serves as a fingerprint for gastric cancer diagnosis. Eur $\mathrm{J}$ Cancer. 2011;47(5):784-91.

26. Liu H, Zhu L, Liu B, Yang L, Meng X, Zhang W, et al. Genome-wide microRNA profiles identify miR-378 as a serum biomarker for early detection of gastric cancer. Cancer letters. 2012;316(2):196-203.

27. Song My, Pan K, Su H, Zhang L, Ma J, Li $\mathrm{Jy}$, et al. Identification of serum microRNAs as novel non-invasive biomarkers for early detection of gastric cancer. PloS one. 2012;7(3):e33608.

28. Sierzega M, Kaczor M, Kolodziejczyk P, Kulig J, Sanak M, Richteret P et al. Evaluation of serum microRNA biomarkers for gastric cancer based on blood and tissue pools profiling: the importance of miR-21 and miR-331. Br J Cancer. 2017;117(2):266-273.

29. Matsushima, $K$, Isomoto $H$, Inoue $N$, Nakayama T, Hayashi T, Nakayama M, et al. MicroRNA signatures in Helicobacter pylori-infected gastric mucosa. Int $\mathrm{J}$ Cancer. 2011;128(2):361-70. 
30. Guo J, Miao Y, Xiao B, Huan R, Jiang Z, Meng D, et al. Differential expression of microRNA species in human gastric cancer versus non-tumorous tissues. J Gastroenterol Hepatol. 2009;24(4):652-7.

31. Kim, Y.-K., Yu J, Han TS, Park S, Namkoong B, Kimet DH, et al. Functional links between clustered microRNAs: suppression of cell-cycle inhibitors by microRNA clusters in gastric cancer. Nucleic Acids Res. 2009;37(5):1672-81.

32. Noto JM, RM, Peek Jr. The role of microRNAs in Helicobacter pylori pathogenesis and gastric carcinogenesis. Front Cell Infect Microbiol. 2012;1:21.

33. Volinia S, Calin GA, Liu C, Ambs S, Cimmino A, Petrocca F, et al. A microRNA expression signature of human solid tumors defines cancer gene targets. Proc Natl Acad Sci U S A. 2006;103(7):2257-61.

34. Libânio D, M Dinis-Ribeiro, P PimentelNunes. Helicobacter pylori and microRNAs: Relation with innate immunity and progression of preneoplastic conditions. World J Clin Oncol. 2015;6(5):111-32.

35. Liu L, Chen Qi, Lai R, Wu X, Wu X, Liu F, et al. Elevated expression of mature miR-21 and miR-155 in cancerous gastric tissues from Chinese patients with gastric cancer. J Biomed Res. 2010;24(3):187-197.

36. Kim SS, Meitner P, Konkin T, Cho YS, MB Resnick, Moss SF, et al. Altered expression of Skp2, c-Myc and p27 proteins but not mRNA after $H$. pylori eradication in chronic gastritis. Modern Pathology. 2006;19:49-58. 